\title{
'White cord syndrome', a rare but disastrous complication of transient paralysis after posterior cervical decompression for severe cervical spondylotic myelopathy and spinal stenosis: A case report
}

\author{
YU-XIN LIAO, SHI-SHENG HE and ZHI-MIN HE \\ Department of Orthopaedics, Shanghai 10th People's Hospital, Tongji University \\ School of Medicine, Shanghai 200072, P.R. China
}

Received December 4, 2019; Accepted August 19, 2020

DOI: $10.3892 /$ etm.2020.9218

\begin{abstract}
Transient paralysis following spinal decompression surgery is a rare but devastating postoperative complication. Spinal cord ischemia-reperfusion injury has been identified as one of the crucial pathogenic factors contributing to the sudden neurological deterioration associated with spinal decompression surgery. 'White cord syndrome' is a characteristic imaging manifestation of spinal cord ischemia-reperfusion injury, referring to high intramedullary signal changes in the sagittal T2-weighted MRI scan with unexplained neurological deficits following surgical decompression. The present study reported on the case of a 51-year old male patient who suffered from acute left limb hemiplegic paralysis following posterior cervical laminectomy decompression for severe cervical spondylotic myelopathy and spinal stenosis, which were caused by ossification of the posterior longitudinal ligament. The patient's neurological function gradually improved after the immediate administration of high-dose methylprednisolone therapy combined with mannitol and neurotrophic drugs. At the 2-month follow-up, the intensity of the spinal cord signal on MRI had almost returned to normal and
\end{abstract}

Correspondence to: Professor Zhi-Min He, Department of Orthopaedics, Shanghai 10th People's Hospital, Tongji University School of Medicine, 301 Yanchang Road, Shanghai 200072, P.R. China

E-mail: spinehe@163.com

Abbreviations: ACCF, anterior cervical corpectomy and fusion; ACDF, anterior cervical decompression and fusion; DCM, degenerative cervical myelopathy; MEP, motor-evoked potential; NASCIS, National Acute Spinal Cord Injury Study; OLF, ossification of the ligamentum flavum; OPLL, ossification of the posterior longitudinal ligament; SSEPs, somatosensory evoked potentials

Key words: white cord syndrome, spinal cord ischemia-reperfusion injury, paralysis, cervical decompression, methylprednisolone the 'white cord syndrome' had disappeared. However, the patient complained of postoperative neck swelling pain caused by cerebrospinal fluid leakage; therefore, an additional cerebrospinal fluid leakage exploration and neoplasty were performed. At 2 weeks after the second surgery, the patient's neck swelling pain was relieved and the area of cerebrospinal fluid leakage was significantly reduced. Despite the low incidence rate, surgeons should be aware of this complication, particularly when treating chronic severe cervical spinal stenosis with anterior or posterior decompression. Once transient paralysis occurs, early diagnosis and interventions are essential to reverse the neurological deficit.

\section{Introduction}

Transient paralysis following surgical decompression is a severe postoperative complication that may occur in patients with chronic severe spinal cord compression (1). Spinal cord ischemia-reperfusion injury has been confirmed as a potential etiology of the associated neurological deterioration. Spinal cord ischemia-reperfusion injury is defined as the sudden expansion of the compressed cord and exposure to a rush in blood supply following decompression surgery, which leads to disruption of the blood-brain barrier and blood-spinal cord barrier, and may ultimately result in acute neurologic dysfunction $(2,3)$. The reported incidence rate of spinal cord ischemia-reperfusion injury following cervical decompression surgery ranges from $2-5.7 \%$, but a higher incidence rate of $14.5 \%$ has been reported for posterior decompressive laminectomy procedures used for thoracic spinal stenosis $(4,5)$. 'White cord syndrome', initially described by Chin et al (2) in 2013, is an imaging feature of spinal cord ischemia-reperfusion injury that is present on postoperative MRI scans. 'White cord syndrome' refers to high intramedullary signal changes observed on sagittal T2-weighted MRI scans in patients with unexplained incomplete paralysis following cervical decompression surgery (2). In the present study, the 9th case of 'white cord syndrome' following cervical decompression surgery according to previous literature, was reported. 

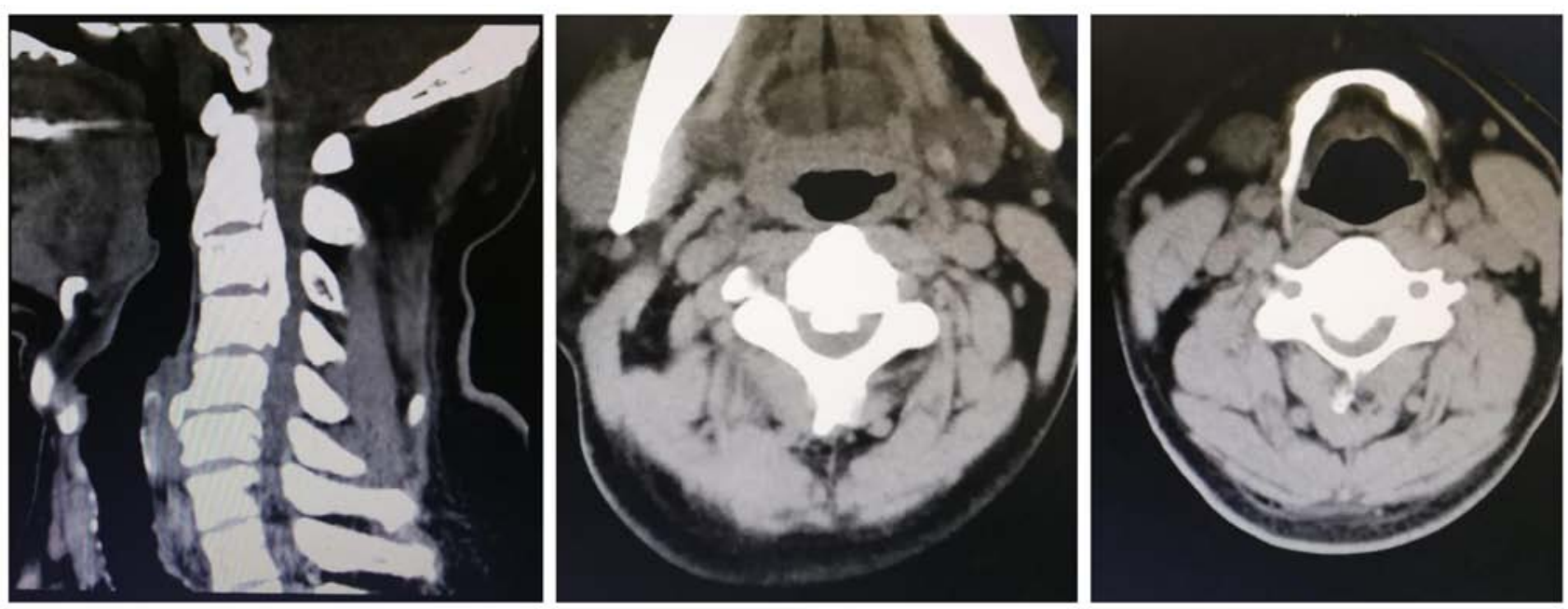

Figure 1. Preoperative cervical CT scan displaying the anterior compression of the spinal cord by OPLL at the C2-C4 levels, which led to severe cervical spinal stenosis. The occupying ratio (calculated as the thickness of OPLL/the anteroposterior diameter of the spine) at the C2/3 and C3/4 levels was $~ 46.7$ and $63 \%$, respectively. OPLL, ossification of posterior longitudinal ligament.

\section{Case report}

A 51-year old male presented with the chief complaint of numbness in the bilateral upper extremities and an unsteady gait for 6 months. Neurological examination indicated that muscle strength in the left upper limb was grade 4/5 from the Medical Research Council Scale (6). Tendon reflexes, including biceps, triceps, radial periosteal, knees and ankles, displayed bilateral hyperreflexia. A positive Babinski sign was also identified bilaterally. The preoperative cervical CT and MRI scans revealed that the spinal cord was anteriorly compressed by ossification of the posterior longitudinal ligament (OPLL) at the C2-C4 levels, which led to severe cervical spinal stenosis (Figs. 1 and 2). On $\mathrm{CT}$, the occupying ratio (calculated as the thickness of OPLL/the anteroposterior diameter of the spinal canal) at the $\mathrm{C} 2 / 3$ and C3/4 levels was 46.7 and 63\%, respectively (Fig. 1).

Due to the difficulty in operative exposure and high risk of cerebrospinal fluid leakage by either anterior cervical decompression and fusion (ACDF) or anterior cervical corpectomy and fusion (ACCF) for high-level cervical spondylotic myelopathy combined with OPLL (C2-C4), the patient underwent C3-C4 posterior laminectomy decompression and $\mathrm{C} 2-\mathrm{C} 5$ fixation with pedicle screws. Intraoperatively, sudden cord expansion occurred after the vertebral plates were completely removed. An intravenous methylprednisolone bolus (500 mg) was immediately administrated. On return to the ward, the patient displayed hemiplegic paralysis and was not able to move the left limb, displaying a muscle strength of grade $1 / 5$ in the lower left extremity and grade $2 / 5$ in the upper left extremity. Reperfusion injury following acute decompression of the severely and chronically compressed spinal cord was highly suspected in the patient; therefore, high-dose methylprednisolone combined with mannitol and neurotrophic drugs were rapidly administered intravenously. The patient received methylprednisolone ( $30 \mathrm{mg} / \mathrm{kg}$ ) over $15 \mathrm{~min}$, followed by a $45-\mathrm{min}$ rest and a 23 -h maintenance infusion $(5.4 \mathrm{mg} / \mathrm{kg} / \mathrm{h})$, according to the National Acute Spinal Cord Injury Study II (NASCIS-2) protocol (7). In addition, omeprazole $(40 \mathrm{mg}$ ) was administered to the patient prior to each dose of methylprednisolone to prevent gastrointestinal bleeding, which may be caused by high-dose methylprednisolone. Furthermore, postoperative $\mathrm{X}$-ray, CT and MRI scans were performed to evaluate the spinal cord function. X-ray and CT scans displayed satisfactory positioning of the pedicle screws (Figs. 3 and 4). However, T2-weighted MRI scans demonstrated a signal of abnormally high intensity in the spinal cord at the C4-C7 levels. In addition, a large-area hyperintense signal was observed behind the epidural at the C3-C6 levels, which indicated cerebrospinal fluid leakage (Fig. 5A). At 12 h post-methylprednisolone treatment, the patient's muscle strength improved by one grade to grade $2 / 5$ in the left lower extremity and to grade $3 / 5$ in the left upper extremity. On postoperative day 5 , the muscle strength in the left limb recovered to grade $4 / 5$, which was the same as the preoperative level. Considering the patient displayed no wound exudation, fever or dizziness, the postoperative cerebrospinal fluid leakage was dynamically observed without treatment. At day 8 post-surgery, the patient was discharged to rehabilitation with hyperbaric oxygen therapy.

At the 2-month follow-up, the patient's neurological status had not deteriorated; however, the patient complained of neck swelling and associated pain following the surgery. MRI scans were repeatedly performed to assess the spinal cord injury and cerebrospinal fluid leakage. The intensity of the spinal cord signal had almost returned to normal at the C4-C7 levels (Fig. 5B); however, the area of cerebrospinal fluid leakage had not decreased, but had expanded to the subcutaneous tissue. The patient was readmitted for an additional cerebrospinal fluid leakage exploration and neoplasty. During the second surgery, an abundance of clear liquid was released when the wound was reopened. A large crevasse was identified on the left side of the dura, close to the pedicle at the $\mathrm{C} 4$ level, and an artificial dura mater was used to repair the crevasse (Fig. S1). At 2 weeks post-surgery, the neck swelling and associated pain was relieved and an MRI scan indicated that the area of cerebrospinal fluid leakage had obviously decreased (Fig. 5C). The patient was then discharged to an inpatient rehabilitation center for physical therapy. At the last 6-month telephone follow-up, his neurological function was stable without neck swelling and associated pain. 

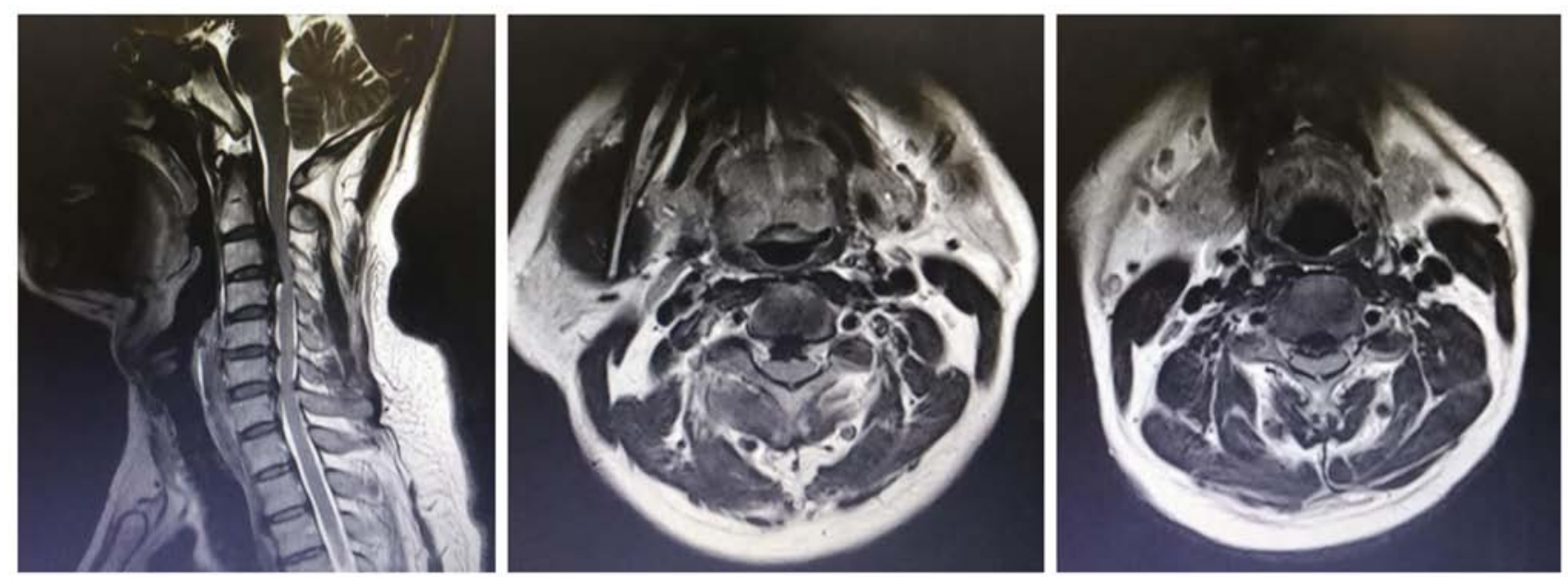

Figure 2. Preoperative cervical MRI scan displaying the compressed spinal cord and severe cervical stenosis at the C2-C4 levels.
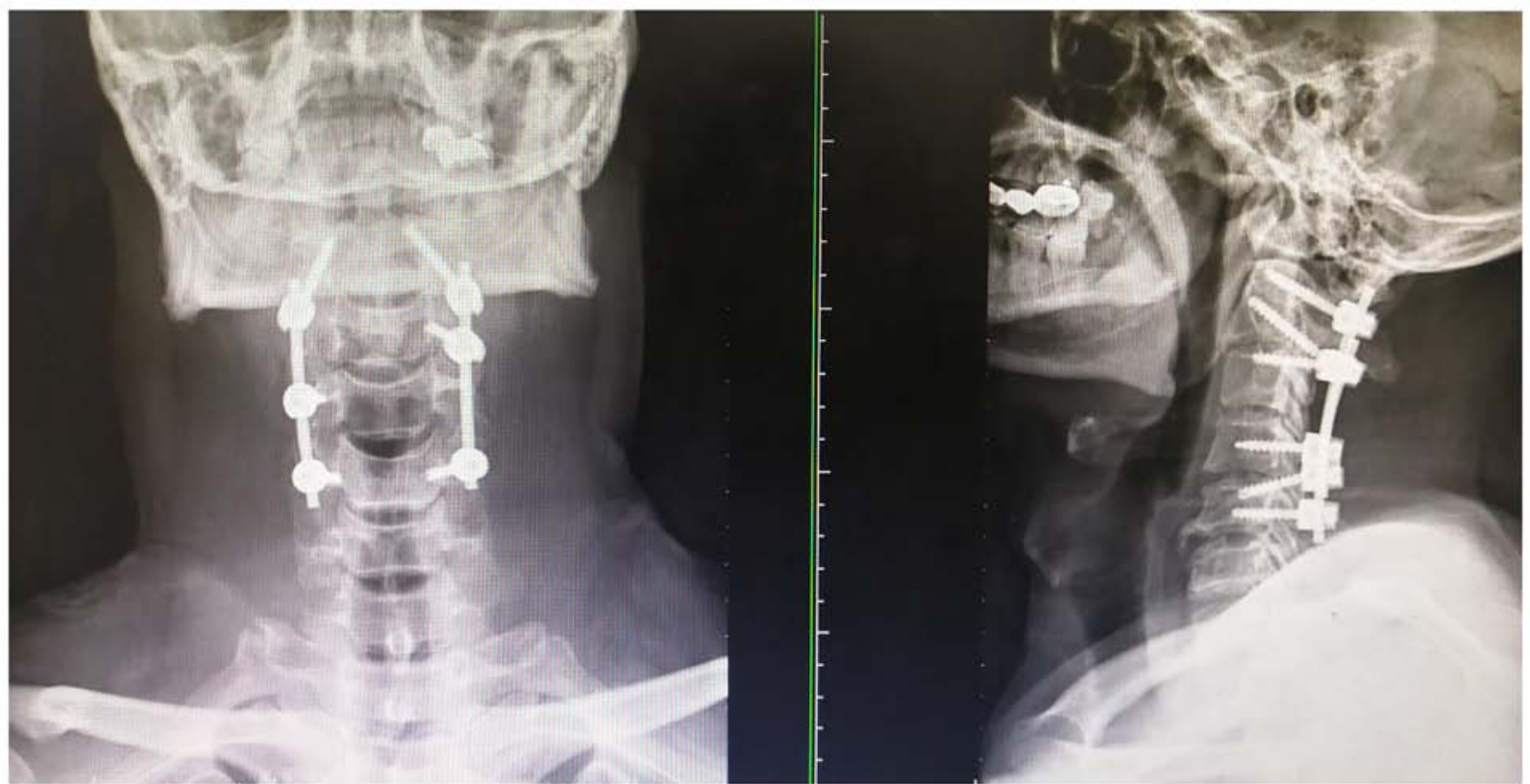

Figure 3. Postoperative X-ray displaying the satisfactory placement of the screws and rods at the C2-C5 levels.

\section{Discussion}

Fixation failure, epidural hematoma, spinal cord edema and ischemia-reperfusion injury are the major factors that contribute to acute paralysis following spinal surgery (8). On postoperative CT and MRI scans, it is possible to identify the position of the implant and epidural hematomas, respectively. Spinal cord edema typically peaks at $48-72 \mathrm{~h}$ post-surgery and symptoms may be relieved by dehydration. Diagnostic criteria for spinal cord ischemia-reperfusion injury primarily include the following: Severe spinal cord compression; surgical decompression; paralysis occurring within $3 \mathrm{~h}$ post-surgery; motor and sensory dysfunction from lower to upper limb; exclusion of other causative factors, including hematoma; and completely or partially restored neurological function by timely high-dose methylprednisolone combined with dehydration and neurotrophic drugs $(9,10)$. Zhang et al $(8)$ reported on three cases where transient paralysis had occurred from 30 min to $4 \mathrm{~h}$ post-ACCF in patients with cervical spondylotic myelopathy. However, no hematoma was identified in the post-operative MRI scans; therefore, spinal cord ischemia-reperfusion injury was suspected. Following administration of high-dose methylprednisolone combined with dehydration and neurotrophic drugs, a full recovery was achieved in two patients. By contrast, the neurological function of one patient was not restored until an additional cervical posterior decompression procedure was performed. The pathogenesis of the patients was identical to that in the case reported by Khan et al (11), who described a 36-year-old male who had developed a delayed neurological deficit on day 3 post-ACDF for C5-6 cervical disc herniation.

Postoperative spinal cord ischemia-reperfusion injury has not only been reported in cervical decompression surgery, but also in posterior decompression for thoracic spinal stenosis caused by ossification of the ligamentum flavum (OLF) and 

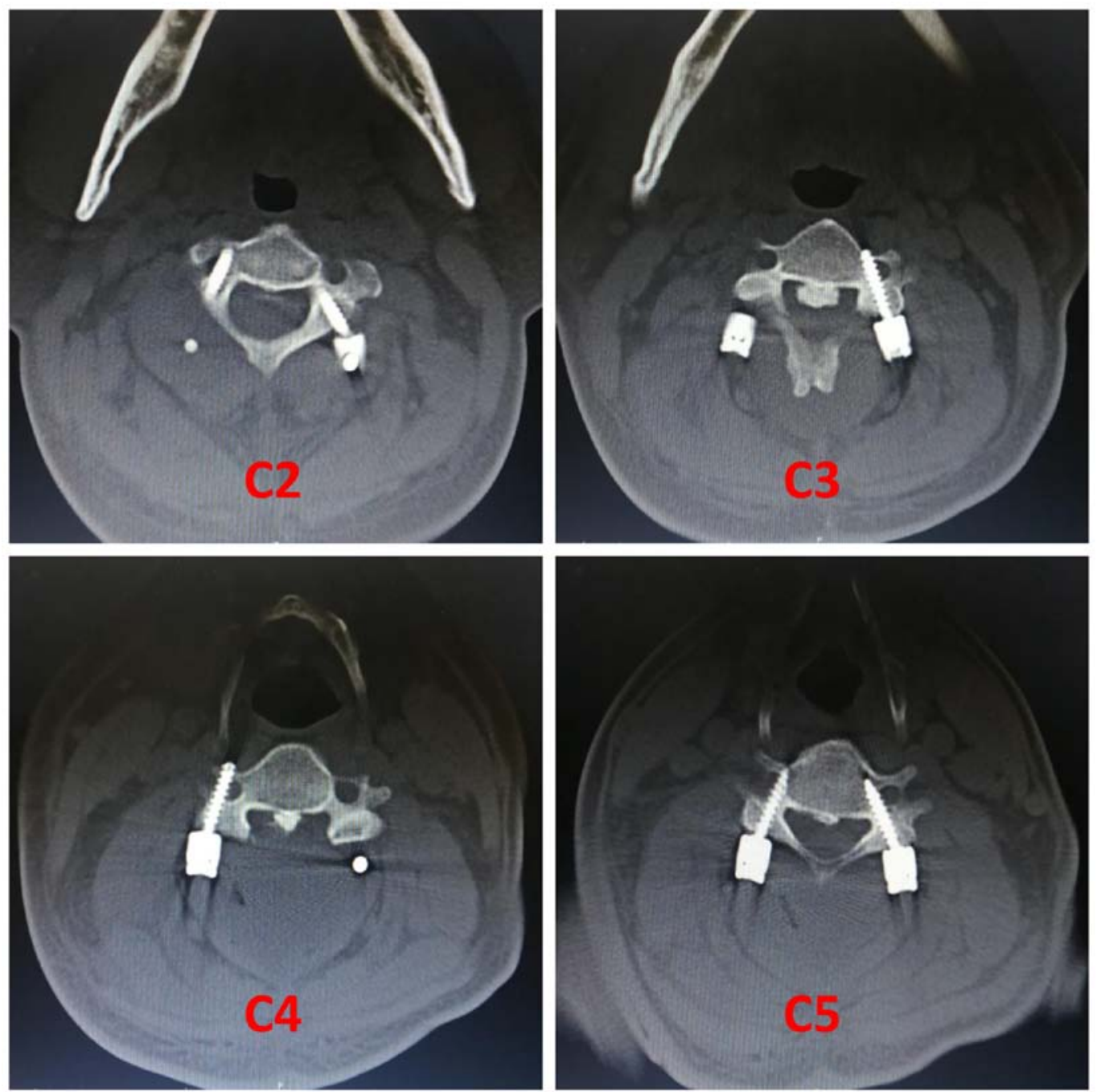

Figure 4. Postoperative CT scans displaying the satisfactory positioning of the pedicle screws at the C2-C5 levels.
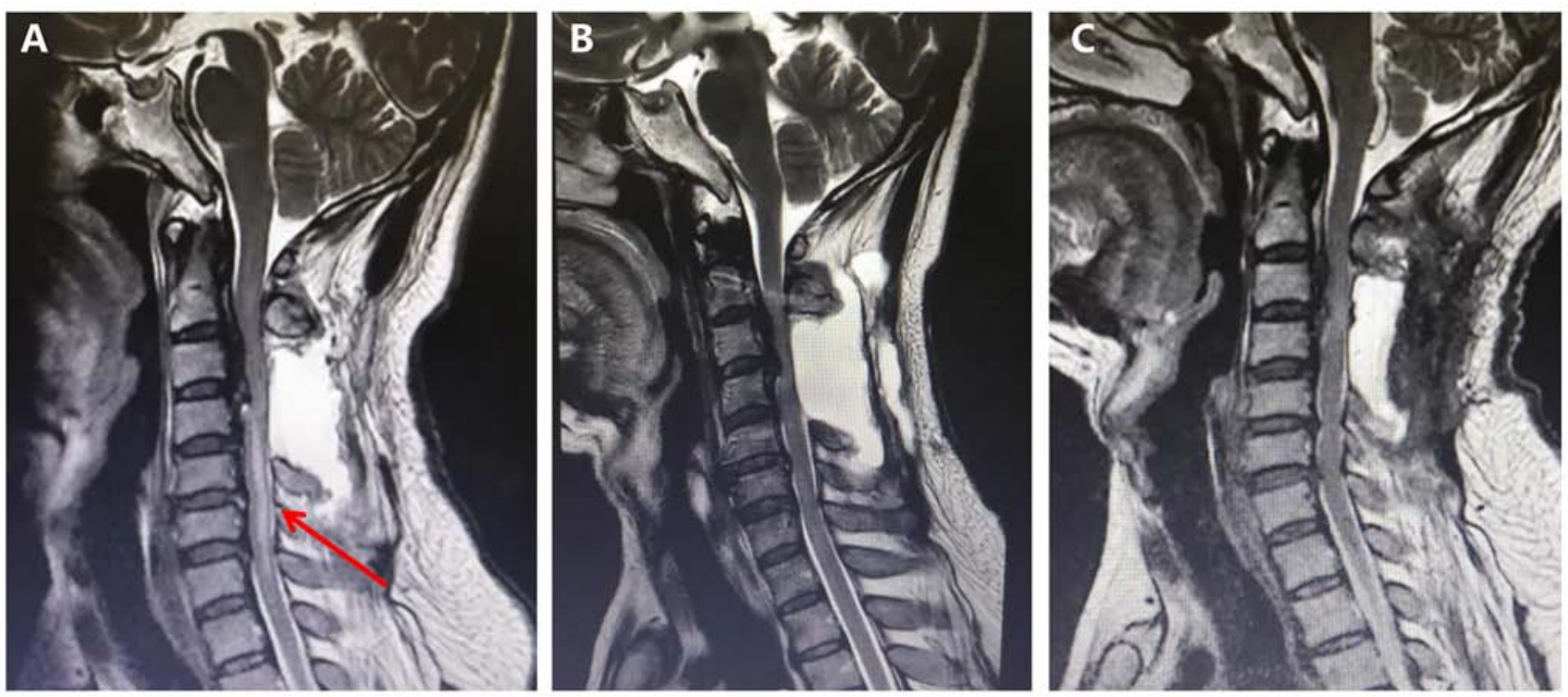

Figure 5. (A) Immediate postoperative sagittal T2-weighted MRI scan displaying a signal of abnormally high intensity in the spinal cord at C4-C7 levels (as indicated by the red arrow). A large-area hyperintense signal was observed behind the epidural at the C3-C6 levels, which indicated cerebrospinal fluid leakage. (B) At the 2-month follow-up, the sagittal T2-weighted MRI scan indicated that the intensity of the spinal cord signal had almost returned to normal at the C4-C7 levels. However, the area of cerebrospinal fluid leakage was not reduced, but had expanded to the subcutaneous tissue. (C) At 2 weeks after the additional cerebrospinal fluid leakage exploration and neoplasty, the sagittal T2-weighted MRI scan indicated that the area of cerebrospinal fluid leakage had obviously decreased. 
OPLL. Yamazaki et al (12) described the case of a 71-year old female who was diagnosed with thoracic myelopathy caused by OPLL and OLF, and underwent a thoracic posterior laminectomy at T10-T11. At $18 \mathrm{~h}$ post-surgery, the patient was suddenly not able to move both lower extremities, displaying of muscle strength of grade $0 / 5$. Despite immediately administering an intravenous bolus dose of methylprednisolone sodium succinate $(1 \mathrm{~g})$ followed by an additional 2-h maintenance infusion of methylprednisolone sodium succinate $(1 \mathrm{~g})$, the patient's motor function did not improve. At $4 \mathrm{~h}$ post-paralysis, the patient received an additional posterior instrumented fusion at T7-L1. At $3 \mathrm{~h}$ after the second surgery, the patient's motor function gradually recovered. At the final follow-up (5 years and 9 months post-surgery), the patient's neurological status was improved and stabilized with grade 4-5/5 muscle strength in both lower extremities. In addition, a high-intensity signal was observed at T10-T11 on the T2-weighted MRI performed 1 week after the second surgery and the signal intensity returned to normal when the patient's neurological function improved. Based on this case, it was recommended that posterior instrumented fusion should be used to reduce postoperative paralysis when posterior decompression for thoracic OLF and OPLL is performed. Similarly, Taher et al (13) reported on three cases with transient neurological deficit without spinal cord injury following decompression for severe thoracic stenosis. The recovery time among the patients ranged from 1-13 months. Ischemia-reperfusion injury was regarded as the major cause of postoperative paralysis. Furthermore, intraoperative electrophysiological monitoring, which changed immediately once paralysis had occurred during surgery, was recommended as a useful tool to avoid neurological deterioration. In addition, Yang et al (14) reported that 10 patients developed delayed neurological deficits following surgical removal of intraspinal meningiomas ( 7 located in the thoracic region and 3 located in the cervical region). The occurrence time in those cases was 3-8 h post-surgery. Postoperative T2-weighted MRI scans revealed high intramedullary signal changes in four patients; however, ischemia-reperfusion injury was also considered to be responsible for the observed deterioration.

The most widely accepted pathophysiological mechanism underlying 'white cord syndrome' is the sudden expansion of the chronically compressed cord and subsequent exposure to a rush of blood supply following decompression surgery, which triggers an inflammatory cascade and releases oxygen free radicals, ultimately leading to neuronal membrane damage $(15,16)$. Chin et al (2) reported the first case of "white cord syndrome' in a 59-year old male with severe $\mathrm{C} 5 / 6$ disc herniation who had undergone ACDF at the $\mathrm{C} 4 / 5$ and $\mathrm{C} 5 / 6$ level. The patient displayed complete loss of somatosensory evoked potentials (SSEPs) during the operation and postoperative C6 incomplete tetraplegia. A large area of high signal intensity was identified in the spinal cord on the postoperative MRI scan, and the term 'white cord syndrome' was used, referring to the radiographic changes. Following on from the aforementioned study, white cord syndrome after anterior or posterior cervical decompression has received increasing attention. Antwi et al (17) reported the case of 'white cord syndrome' in a 68 -year-old male who had developed acute hemiparesis on the left side following $\mathrm{C} 4-\mathrm{C} 7$ posterior laminectomy decompression in 2018. Also in 2018, Vinodh et al (3) reported similar findings in a 51-year-old female who was diagnosed with a metastatic intraspinal tumor and presented with quadriplegia following posterior cervical laminectomy and fusion for tumor resection. Papaioannou et al (18) reported the case of 'white cord syndrome' in a 79-year old male who had undergone C4-C6 ACDF 2 years previously because of cervical spondylotic myelopathy. The patient suffered from C6 incomplete paraplegia after the second C3-C6 posterior decompression surgery. Papaioannou et al (18) further indicated that differential diagnoses of 'white cord syndrome' included iatrogenic trauma leading to cerebrospinal fluid leakage, pseudo meningocele, cerebrovascular injury and demyelinating disease. According to the latest literature review of 'white Cord syndrome' by Mathkour et al (16), there are a total of 7 cases, including 2 cases after ACDF surgery and 5 cases following posterior cervical decompression surgery. Recently, another case of 'white Cord syndrome' after ACDF is reported by Jun et al (19). Consistent with previous cases, high intramedullary signals were also observed on the postoperative sagittal T2-weighted MRI scan and the neurologic deficit was reversed by immediate high-dose steroid administration in the case reported in the present study. Furthermore, although the patient displayed cerebrospinal fluid leakage, 'white cord syndrome' was diagnosed on the basis of the observation that the hyperintense signal in the spinal cord disappeared, although cerebrospinal fluid leakage was still present at the 2-month follow-up, prior to the cerebrospinal fluid leakage exploration and neoplasty.

Ever since positive results of the NASCIS- 2 protocol were reported in 1990 (7), the use of high-dose methylprednisolone for the treatment of acute spinal cord injury has been widely accepted worldwide. According to the guidelines published in 2017, treatment strategy of 24-h infusion of high-dose methylprednisolone is recommended for patients within $8 \mathrm{~h}$ following injury (20). To identify the exact pathological mechanisms underlying ischemia-reperfusion injury caused by decompression, a number of studies established a mouse model of degenerative cervical myelopathy (DCM). Yang et al (1) reported that surgical decompression increased the expression of inflammatory cytokines, including TNF- $\alpha$ and IL-1 $\beta$, in an animal model of chronic severe spinal cord compression. Similarly, Vidal et al (21) suggested that decompression led to a 1.5- to 2-fold increase in expression levels of inflammatory cytokines in a mouse model of DCM. It was also reported that delayed decompression aggravated ischemia-reperfusion injury, resulting in decreased neurological improvement compared with earlier intervention. In another study, perioperative methylprednisolone treatment (30 min prior to decompression and 2 weeks after decompression at one dose of $30 \mathrm{mg} / \mathrm{kg}$ ) reduced neurological deterioration caused by ischemia-reperfusion injury via its anti-inflammatory effect in a mouse model of DCM (22). In animal models, the levels of inflammatory cytokines such as TNF- $\alpha$ and IL-1 $\beta$ increased when decompression-associated spinal cord ischemia-reperfusion injury occurred, indicating that inflammatory cytokines may be considered as an important evaluation indicator of decompression-associated spinal cord ischemia-reperfusion injury $(1,21,22)$.

In the case described in the present study, signals of abnormally high intensity in the spinal cord were detected on 
the postoperative sagittal T2-weighted MRI scan once acute paralysis had occurred, which displayed similarities to the aforementioned cases of 'white cord syndrome' $(2,3,17,18)$. The neurological function of the patient gradually improved with the administration of high-dose methylprednisolone therapy, according to the NASCIS-2 protocol. Although cerebrospinal fluid leakage occurred in the patient, transient paralysis was not attributed to this iatrogenic injury because the muscle strength in the left limb had recovered to normal prior to the cerebrospinal fluid leakage exploration and neoplasty. A limitation of the management of the case reported in the present study was that intraoperative neurophysiological monitoring was not conducted to evaluate SSEPs and motor-evoked potential (MEP) alterations during the operation.

Despite the low incidence rate of 'white cord syndrome', spinal surgeons should take action to reduce the risk of this disastrous complication when performing posterior cervical decompression surgery for chronic severe spinal cord compression. First, preoperative remote ischemic preconditioning, consisting of three cycles of upper right limb ischemia for $5 \mathrm{~min}$ with 5 -min intervals of reperfusion between each cycle, may prevent spinal cord ischemic injury by reducing neuro-specific enolase and S-100B expression after surgery. Enolase and S-100B are two useful diagnostic biomarkers for spinal cord injury (4). Furthermore, intraoperative neurophysiological monitoring is an effective tool to evaluate SSEP and MEP alterations, and to indicate whether spinal cord injury has occurred during surgery (23). Finally, posterior cervical laminectomies were performed in all reported cases of 'white cord syndrome' following posterior decompression surgery $(3,17,18)$, including the present case report. In all cases, it may be recommended to opt for posterior cervical laminoplasty instead of posterior cervical laminectomy to avoid excessive expansion of the spinal cord and to reduce the risk of ischemia-reperfusion injury.

Cerebrospinal fluid leak, as a postoperative complication of decompression surgery, occurred in the case reported in the present study. Small and invisible dura defects caused by bipolar coagulation were not identified intraoperatively and dura repair was not performed until the second revision surgery. Cerebrospinal fluid leak repair primarily involves primary or delayed repair (with a non-absorbable suture or fibrin sealant) and lumbar drainage $(15-20 \mathrm{ml} / \mathrm{h}$ for 5 days) (24). Woodroffe et al (24) analyzed the clinical prognosis of 124 patients with cerebrospinal fluid leak using different treatments. The successful repair rate was $73.4 \%$ (47/64) in the primary repair group and $87.8 \%$ (43/49) in the lumbar drainage group. Furthermore, $39.5 \%$ of the patients who had not received primary repair and lumbar drainage procedures were subjected to revision surgery. Delayed repair was also associated with increased hospital stays and higher risk of infection compared with primary repair. Therefore, the analysis emphasized the importance of intraoperative prompt identification and strongly recommended that primary repair of dura defects should be combined with lumbar drainage whenever possible. In another study, Fang et al (25) proposed two treatment strategies for preventing cerebrospinal fluid leak: i) Direct suture with a dural substitute material; and ii) reducing the subarachnoid fluid pressure or increasing the epidural space pressure.
In conclusion, transient paralysis following surgical decompression for chronic severe spinal cord compression is a catastrophic postoperative complication. Spinal cord ischemia-reperfusion injury, which has an imaging feature of high intramedullary signal changes on the postoperative sagittal T2-weighted MRI scan and therefore referred to as 'white cord syndrome', has been considered as an important pathogenic factor contributing to neurological deterioration. Regardless of its low incidence rate, surgeons should be aware of this devastating complication, particularly when treating long-term and severe spinal stenosis caused by OPLL and OLF. Once transient paralysis occurs, early diagnosis and high-dose methylprednisolone therapy are essential to reverse the associated neurological deficits.

\section{Acknowledgements}

Not applicable.

\section{Funding}

No funding was received.

\section{Availability of data and materials}

All data generated or analyzed during the present study are included in this published article and its supplementary information files.

\section{Authors' contributions}

YXL designed the study, collected clinical data and drafted the manuscript. SSH and ZMH designed the study and critically revised the manuscript. All of the authors read and approved the final manuscript.

\section{Ethics approval and consent to participate}

The study was approved by the Ethics Committee of the Shanghai 10th People's Hospital (SHSY-IEC-4.1/20-17/01). The patient signed the informed consent form.

\section{Patient consent for publication}

Written informed consent for the publication of patient data and accompanying images was obtained.

\section{Competing interests}

The authors declare that they have no competing interests.

\section{References}

1. Yang $\mathrm{T}$, Wu L, Wang $\mathrm{H}$, Fang J, Yao $\mathrm{N}$ and $\mathrm{Xu}$ Y: Inflammation level after decompression surgery for a rat model of chronic severe spinal cord compression and effects on ischemia-reperfusion injury. Neurol Med Chir (Tokyo) 55: 578-586, 2015.

2. Chin KR, Seale J and Cumming V: 'White cord syndrome' of acute tetraplegia after anterior cervical decompression and fusion for chronic spinal cord compression: A case report. Case Rep Orthop 2013: 697918, 2013. 
3. Vinodh VP, Rajapathy SK, Sellamuthu P and Kandasamy R White cord syndrome: A devastating complication of spinal decompression surgery. Surg Neurol Int 9: 136, 2018.

4. Hu S, Dong HL, Li YZ, Luo ZJ, Sun L, Yang QZ, Yang LF and Xiong L: Effects of remote ischemic preconditioning on biochemical markers and neurologic outcomes in patients undergoing elective cervical decompression surgery: A prospective randomized controlled trial. J Neurosurg Anesthesiol 22: 46-52, 2010.

5. Young WF and Baron E: Acute neurologic deterioration after surgical treatment for thoracic spinal stenosis. J Clin Neurosci 8: 129-132, 2001.

6. Vanpee G, Hermans G, Segers J and Gosselink R: Assessment of limb muscle strength in critically ill patients: A systematic review. Crit Care Med 42: 701-711, 2014.

7. Bracken MB, Shepard MJ, Collins WF, Holford TR, Young W, Baskin DS, Eisenberg HM, Flamm E, Leo-Summers L, Maroon J, et al: A randomized, controlled trial of methylprednisolone or naloxone in the treatment of acute spinal-cord injury. Results of the Second National acute spinal cord injury study. N Engl J Med 322: 1405-1411, 1990.

8. Zhang JD, Xia Q, Ji N, Liu YC, Han Y and Ning SL: Transient paralysis shortly after anterior cervical corpectomy and fusion. Orthop Surg 5: 23-28, 2013.

9. Jiang X, Shi E, Nakajima Y and Sato S: Postconditioning, a series of brief interruptions of early reperfusion, prevents neurologic injury after spinal cord ischemia. Ann Surg 244: 148-153, 2006.

10. Back MR, Bandyk M, Bradner M, Cuthbertson D, Johnson BL, Shames ML and Bandyk DF: Critical analysis of outcome determinants affecting repair of intact aneurysms involving the visceral aorta. Ann Vasc Surg 19: 648-656, 2005.

11. Khan MF, Jooma R, Hashmi FA and Raghib MF: Delayed spinal cord infarction following anterior cervical surgical decompression. BMJ Case Rep 2017: bcr2017219863, 2017.

12. Yamazaki M, Koda M, Okawa A and Aiba A: Transient paraparesis after laminectomy for thoracic ossification of the posterior longitudinal ligament and ossification of the ligamentum flavum. Spinal Cord 44: 130-134, 2006.

13. Taher F, Lebl DR, Cammisa FP, Pinter DW, Sun DY and Girardi FP: Transient neurological deficit following midthoracic decompression for severe stenosis: A series of three cases. Eur Spine J 22: 2057-2061, 2013.

14. Yang T, Wu L, Deng X, Yang C, Zhang Y, Zhang D and Xu Y: Delayed neurological deterioration with an unknown cause subsequent to surgery for intraspinal meningiomas. Oncol Lett 9: 2325-2330, 2015.

15. Wiginton J IV, Brazdzionis J, Mohrdar C, Sweiss R and Lawandy S: Spinal cord reperfusion injury: Case report, review of the literature, and future treatment strategies. Cureus 11: e5279, 2019.
16. Mathkour M, Werner C, Riffle J, Scullen T, Dallapiazza RF, Dumont A and Maulucci C: Reperfusion 'White Cord' syndrome in cervical spondylotic myelopathy: Does mean arterial pressure goal make a difference? Additional case and literature review. World Neurosurg 137: 194-199, 2020.

17. Antwi P, Grant R, Kuzmik G and Abbed K: 'White Cord Syndrome' of acute hemiparesis after posterior cervical decompression and fusion for chronic cervical stenosis. World Neurosurg 113: 33-36, 2018.

18. Papaioannou I, Repantis T, Baikousis A and Korovessis P: Late-onset 'White Cord Syndrome' in an elderly patient after posterior cervical decompression and fusion: A case report. Spinal Cord Ser Cases 5: 28, 2019.

19. Jun DS, Baik JM and Lee SK: A case report: White cord syndrome following anterior cervical discectomy and fusion: Importance of prompt diagnosis and treatment. BMC Musculoskelet Disord 21: 157,2020

20. Fehlings MG, Wilson JR, Tetreault LA, Aarabi B, Anderson P, Arnold PM, Brodke DS, Burns AS, Chiba K, Dettori JR, et al: A Clinical practice guideline for the management of patients with acute spinal cord injury: Recommendations on the Use of methylprednisolone sodium succinate. Global Spine J 7 (3 Suppl): S203-S211, 2017.

21. Vidal PM, Karadimas SK, Ulndreaj A, Laliberte AM, Tetreault L, Forner S, Wang J, Foltz WD and Fehlings MG: Delayed decompression exacerbates ischemia-reperfusion injury in cervical compressive myelopathy. J CI Insight 2: e92512, 2017.

22. Vidal PM, Ulndreaj A, Badner A, Hong J and Fehlings MG: Methylprednisolone treatment enhances early recovery following surgical decompression for degenerative cervical myelopathy without compromise to the systemic immune system. J Neuroinflammation 15: 222, 2018.

23. Sutter M, Eggspuehler A, Jeszenszky D, Kleinstueck F, Fekete TF, Haschtmann D, Porchet F and Dvorak J: The impact and value of uni- and multimodal intraoperative neurophysiological monitoring (IONM) on neurological complications during spine surgery: A prospective study of 2728 patients. Eur Spine J 28: 599-610, 2019.

24. Woodroffe RW, Nourski KV, Helland LC, Walsh B, Noeller J, Kerezoudis P and Hitchon PW: Management of iatrogenic spinal cerebrospinal fluid leaks: A cohort of 124 patients. Clin Neurol Neurosurg 170: 61-66, 2018.

25. Fang Z, Tian R, Jia YT, Xu TT and Liu Y: Treatment of cerebrospinal fluid leak after spine surgery. Chin J Traumatol 20: 81-83, 2017. 